\title{
Strategies for Management of Relapsed or Refractory Hodgkin Lymphoma
}

\author{
Presented by Leo I. Gordon, MD
}

\section{Abstract}

The advent of effective therapies has improved outcomes for those with newly diagnosed Hodgkin lymphoma (HL), with a resulting cure rate of at least $80 \%$. However, with limited data on therapeutic options in the setting of advanced disease, individualized treatment is recommended, and potential long-term effects of therapy remain a key consideration. At the NCCN 22nd Annual Conference, Dr. Leo I. Gordon explored strategies for systemic therapy in the relapsed or refractory setting, focusing primarily on the standard of high-dose therapy/ autologous stem cell rescue, the CD30-targeted antibody drug conjugate brentuximab vedotin, and checkpoint inhibition.

J Natl Compr Canc Netw 2017;15(5.5):716-718

“T here are a number of salvage chemotherapy regimens in relapsed or refractory [R/R] Hodgkin lymphoma [HL]," revealed Leo I. Gordon, MD, Abby and John Friend Professor of Cancer Research and Professor of Medicine, Division of Hematology/Oncology, Robert H. Lurie Comprehensive Cancer Center. "The concept here is to use a regimen that achieves as close to a complete response [CR] as possible," he added. Dr. Gordon is Vice Chair of the NCCN Non-Hodgkin's Lymphomas Panel and a member of the NCCN Hodgkin Lymphoma Panel.

To begin, several prognostic factors should be considered in R/R HL, including time to relapse ( $<3$ months suggests primary refractory disease; $3-12$ months indicates early relapse), advanced disease stage, and poor performance status. Other negative factors include a low albumin level, anemia, age, lymphocytopenia, extranodal disease, and disease status at transplant.

According to the NCCN Clinical Practice Guidelines in Oncology (NCCN Guidelines), second-line systemic therapy is appropriate for any patient with

Presented by Leo I. Gordon, MD, Division of Hematology/Oncology, Northwestern University Feinberg School of Medicine, Robert H. Lurie Comprehensive Cancer Center of Northwestern University, Chicago, Illinois.

Dr. Gordon has disclosed that he has no financial interests, arrangements, affiliations, or commercial interests with the manufacturers of any products discussed in this article or their competitors.

Correspondence: Leo I. Gordon, MD, Robert H. Lurie Comprehensive Cancer Center, Division of Hematology/Oncology, 676 North St. Clair, Suite 850, Chicago, IL 60611. E-mail: I-gordon@northwestern.edu relapsed disease, regardless of the length of initial remission. Therapy may be given before high-dose therapy/autologous stem cell rescue (HDT/ASCR), with or without radiation therapy.

"The idea of using radiation therapy alone is not a curative option, but a very reasonable palliative approach," according to Dr. Gordon. In selected patients who have long disease-free intervals and other favorable features, selection of second-line therapy should be individualized, based on NCCN recommendations. Dr. Gordon stressed the importance of performing a biopsy to confirm suspected relapse.

\section{Transplant Remains the Standard Approach}

HDT/ASCR remains the best treatment approach for patients with $\mathrm{R} / \mathrm{R}$ classical $\mathrm{HL}$, according to the NCCN Guidelines. There are several salvage chemotherapy regimens, and Dr. Gordon noted that the most commonly used one is either ICE (ifosfamide, carboplatin, etoposide) or DHAP (dexamethasone, high-dose cytarabine, cisplatin). However, he admitted, data exist only on a relatively small number of patients.

High-dose chemotherapy and transplantation remain the standard approach in the $\mathrm{R} / \mathrm{R}$ disease setting, and this recommendation is based on an older study by Schmitz et al. ${ }^{1}$ In this randomized trial, the high-dose regimen consisted of BEAM (carmustine, etoposide, cytarabine, melphalan) in combination with autologous 
hematopoietic stem cell transplantation (SCT). "Clearly, there was an improvement with high-dose chemotherapy and transplant compared with conventional chemotherapy," Dr. Gordon noted. With this approach, approximately $60 \%$ of patients with chemosensitive first relapse of HL were free from treatment failure-a "striking difference in outcomes" compared with patients with chemorefractory disease before transplant.

"For patients who are failing to achieve complete or very good partial remission," suggested Dr. Gordon, "one should begin considering allogeneic transplant."

The benefits of allogeneic transplant in chemotherapy sensitive patients before transplant were confirmed in another study, which used a low-intensity regimen of fludarabine and melphalan. ${ }^{2}$ In this population of 92 heavily pretreated patients, allogeneic SCT resulted in long-term progression-free survival (PFS). For the allografted population, nonrelapse mortality at approximately 4 years was $19 \%$ (at 100 days, it was $8 \%$ ). "It is important to look beyond 1 year to see the late effects of allogeneic transplant," Dr. Gordon urged.

\section{Brentuximab Vedotin}

Other therapies target the hepatocyte growth factorregulated tyrosine kinase substrate (HRS) receptors, such as CD30, CD40, Trail (Apo2L), interleukin-13, and CD80 (Table 1).

Maintenance therapy with the CD30-targeted antibody drug conjugate brentuximab vedotin after HDT/ASCR is included as an option in the NCCN Guidelines for patients with primary refractory disease. This recommendation is based on the randomized phase III AETHERA trial, ${ }^{3}$ which showed that early consolidation with brentuximab vedotin after autologous SCT improved PFS in patients with HL who had high-risk factors for relapse or disease progression after transplantation.

"This study led to the approval of brentuximab vedotin in the maintenance setting," mentioned Dr. Gordon. "If you look out to 40 months and beyond, these curves [those free of progressive disease or death] are starting to come together," suggesting that there was less of a difference over time.

A closer look at the study results showed that PET scan can be a predictive factor in the use of this agent. "In patients who had a negative PET scan before transplant, the difference between brentuximab vedotin and placebo is negligible," Dr. Gordon revealed. He offered the following suggestion for considering brentuximab vedotin in the real-world clinical setting: "For patients whose disease might not be in remission going into transplant or who are not clearly in remission after transplant, there is an advantage to giving another active agent in HL. But for those who may be at high risk but still were PET-negative going into transplant, it [the benefit of another agent] isn't as clear to me."

\section{Checkpoint Inhibition}

Dr. Gordon focused his discussion primarily on 2 recent studies with the checkpoint inhibitor nivolum$\mathrm{ab}$ in advanced disease.

In the first trial by Ansell et al, ${ }^{4} 23$ heavily treated patients with $\mathrm{R} / \mathrm{R}$ HL received nivolumab, and $>75 \%$ of patients were enrolled on the study after a relapse following allogeneic SCT. The overall response rate was $87 \%$, which Dr. Gordon called "fairly exciting data in a small number of patients, most of whom were naïve to brentuximab vedotin." However, he noted, there were few CRs (17\%).

\begin{tabular}{|c|}
\hline Targeting HRS receptors \\
\hline CD30 \\
\hline CD40 \\
\hline Trail (Apo2L) and receptors \\
\hline Interleukin-13 and receptors \\
\hline CD80 (B7.1) \\
\hline $\begin{array}{l}\text { Targeting intracellular survival pathways with small } \\
\text { molecules }\end{array}$ \\
\hline Histone deacetylase (HDAC) inhibitors \\
\hline $\mathrm{PI3K} / \mathrm{Akt} / \mathrm{mTOR}$ \\
\hline Nuclear factor-kappa B (NF-kB) \\
\hline Heat shock protein 90 \\
\hline Targeting the microenvironment \\
\hline Rituximab \\
\hline Lenalidomide \\
\hline $\begin{array}{l}\text { Auto LMP2-cytotoxic T cells for Epstein Barr } \\
\text { virus-positive disease }\end{array}$ \\
\hline
\end{tabular}




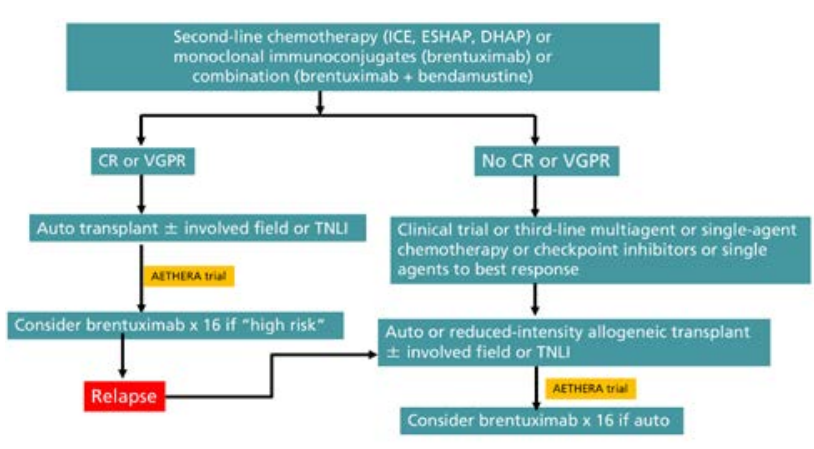

Figure 1. Management of recurrent/refractory Hodgkin lymphoma. Abbreviations: auto, autologous; $\mathrm{CR}$, complete response; DHAP, dexamethasone/cisplatin/high-dose cytarabine; ESHAP, etoposide/ methylprednisolone/high-dose cytarabine/cisplatin; ICE, ifosfamide/ carboplatin/etoposide; VGPR, very good partial response.

In the follow up multicenter phase II trial by Younes et al (CheckMate 205), ${ }^{5}$ the use of nivolumab resulted in frequent responses in patients with recurrent classical HL whose disease had failed to respond to allogeneic SCT and had either relapsed after or failed to respond to brentuximab vedotin. Again, the $\mathrm{CR}$ rates were disappointing, and there was a falloff in PFS at approximately 6 months. "Clearly, we need to do better," Dr. Gordon acknowledged. "It may be that with immunotherapy, our holy grail of CR may not have the same meaning as it has traditionally been used," he added.
Dr. Gordon concluded his presentation with a look at 2 studies exploring the role of allogeneic transplant in the era of programmed cell death protein-1 (PD-1) blockade in classical HL. ${ }^{6,7}$ The first study asked whether allogeneic transplant can safely be performed after PD-1 blockade. According to the findings of a group from Dana-Farber Cancer Institute, ${ }^{6}$ the answer seems to be yes, with a "fairly acceptable" incidence of relapse reported for a highrisk group of patients with $\mathrm{R} / \mathrm{R}$ disease.

However, an increased risk of early immune toxicity is concerning with this therapeutic strategy, and might reflect long-lasting immune alterations triggered by a prior PD- 1 blockade. ${ }^{6}$ In this study, $>70 \%$ of patients had graft-versus-host disease (GVHD). "We have to be very cautious about routinely performing an allogeneic SCT after checkpoint inhibitor therapy," warned Dr. Gordon.

The second trial asked whether PD-1 blockade could be safely performed after allogeneic SCT. Again, the answer seems to be yes: in this small study of 12 patients with R/R HL, nivolumab was effective, with 7 of 8 evaluated patients experiencing a response (3 CRs and 4 partial responses). However, again, acute GVHD was observed in 2 patients after 1 to 2 injections of nivolumab.

A summary of the management algorithm for patients with R/R classical HL is presented in Figure 1.

\section{References}

1. Schmitz N, Pfistner B, Sextro $M$, et al. Aggressive conventional chemotherapy compared with high-dose chemotherapy with autologous haemopoietic stem-cell transplantation for relapsed chemosensitive Hodgkin's disease: a randomised trial. Lancet 2002;359:2065-2071.

2. Sureda A, Canals C, Arranz R, et al. Allogeneic stem cell transplantation after reduced intensity conditioning in patients with relapsed or refractory Hodgkin's lymphoma: results of the HDR-ALLO study. Haematologica 2012;97:310-317.

3. Moskowitz $\mathrm{CH}$, Nademanee A, Masszi T, et al. Brentuximab vedotin as consolidation therapy after autologous stem-cell transplantation in patients with Hodgkin's lymphoma at risk of relapse or progression (AETHERA): a randomised, double-blind, placebo-controlled, phase 3 trial. Lancet 2015;385:1853-1862.

4. Ansell SM, Lesokhin AM, Borrello I, et al. PD-1 blockade with nivolumab in relapsed or refractory Hodgkin's lymphoma. N Engl J Med 2015;372:311-319.

5. Younes A, Santoro A, Shipp M, et al. Nivolumab for classical Hodgkin's lymphoma after failure of both autologous stem-cell transplantation and brentuximab vedotin: a multicentre, multicohort, single-arm phase 2 trial. Lancet Oncol 2016;17:1283-1294.

6. Merryman RW, Kim HT, Zinzani PL, et al. Safety and efficacy of allogeneic hematopoietic stem cell transplant after PD-1 blockade in relapsed/ refractory lymphoma. Blood 2017;129:1380-1388.

7. Herbaux C, Gauthier J, Brice P, et al. Nivolumab is effective and reasonably safe in relapsed or refractory Hodgkin's lymphoma after allogeneic hematopoietic cell transplantation: a study from the Lysa and SFGM-TC [abstract]. Blood 2015;126:Abstract 3979. 